\title{
Role of boundary conditions in dynamic studies of nuclear giant resonances and collisions
}

\author{
P.-G. Reinhard, ${ }^{1,2}$ P. D. Stevenson, ${ }^{2,3}$ D. Almehed, ${ }^{3}$ J. A. Maruhn, ${ }^{2,4}$ and M. R. Strayer ${ }^{5}$ \\ ${ }^{1}$ Institut für Theoretische Physik II, Universität Erlangen-Nürnberg, Staudtstrasse 7, D-91058 Erlangen, Germany \\ ${ }^{2}$ Joint Institute for Heavy-Ion Research, Oak Ridge National Laboratory, P. O. Box 2008, Oak Ridge, Tennessee 37831, USA \\ ${ }^{3}$ Department of Physics, University of Surrey, Guildford, Surrey GU2 7XH, United Kingdom \\ ${ }^{4}$ Institut für Theoretische Physik, Universität Frankfurt, Robert-Mayer-Strasse 8-10, D-60325 Frankfurt am Main, Germany \\ ${ }^{5}$ Physics Division, Oak Ridge National Laboratory, Oak Ridge, Tennessee 37831-6373, USA
}

(Received 8 June 2005; revised manuscript received 3 January 2006; published 28 March 2006)

\begin{abstract}
Absorbing boundary conditions are often employed in time-dependent mean-field calculations to cope with the problem of emitted particles which would otherwise return back onto the system and falsify the dynamical evolution. We scrutinize two widely used methods, imaginary potentials and gradual attenuation by a mask function. To that end, we consider breathing oscillations of a ${ }^{16} \mathrm{O}$ nucleus computed on a radial onedimensional grid in coordinate space. The most critical test case is the computation of resonance spectra in the (linear) domain of small amplitude motion. Absorbing bounds turn out to provide a reliable alternative to fully fledged continuum random phase approximation (RPA) calculations, although rather large absorbing bounds are required to simulate reliably well continuum conditions. We also investigate the computation of observables in the nonlinear domain. This regime turns out to be less demanding. Smaller absorbing margin suffice to achieve the wanted absorption effect.
\end{abstract}

DOI: 10.1103/PhysRevE.73.036709

PACS number(s): 02.70.- c, 21.60.Jz

\section{INTRODUCTION}

A starting-point, from a microscopic point of view, to the solution of quantum many-body problems is the mean-field approach. Depending on the system of interest, the mean field might take the form of, for example, Hartree-Fock, a density functional or the local density approximation. Each of these approaches can include time-dependence to yield time-dependent mean field (TDMF) equations. A code which solves TDMF equations gives one access to a laboratory in a computer, in which ones many-body objects of interest (e.g., nuclei, atoms, molecules, or metal clusters) can interact with each other or with external fields.

Practical realizations of TDMF are often implemented using coordinate space grids, with the coupled nonlinear TDMF equations solved using finite difference techniques. A particular numerical issue that arises in such codes is to deal with the boundaries. For many dynamical processes of interest, particles will be emitted from the system, which means that the wavefunctions representing them will reach the boundary of the grid. Typical boundary conditions, which can be implemented without computational expense are reflecting and periodic boundary conditions. In the first case, outgoing particle flux is reflected back into the system, and in the second it reappears on the other side of the grid. This is essentially never the physical situation one is interested in, and will lead to artefacts in the physical results of the calculation. In the case of nuclear heavy-ion collisions, for example, the interference with the reflected particle flux disturbs the motion of the reaction partners.

The present work seeks to explore some of the problems associated with boundary conditions in TDMF applications. As a test case, we consider the breathing dynamics of ${ }^{16} \mathrm{O}$ in various dynamical regimes. In the linear regime, we scruti- nize the description of nuclear giant resonance spectra. In the nonlinear regime, we check particle emission as one typical observable. To highlight the importance of absorbing bounds, and the wide applicability of the time-dependent mean-field method we also consider the case of two colliding ${ }^{16} \mathrm{O}$ nuclei.

Nuclear giant resonances are highly collective excitations which carry a lot of interesting information about nuclear structure and dynamics. For a recent review of theoretical and experimental work on giant resonances in general, see [1]. The resonances occur above neutron emission threshold, in all regions of the nuclear chart, and at various channels of multipolarity, $L$, spin, $S$ and isospin $T$. Being above threshold, all giant resonances are heavily damped. Their width arises in three ways [2,3]: The Landau width, due to fragmentation, i.e., the spreading of the initial multipole excitation over the many single-particle excitations in energetic vicinity (giant resonances are coherent sums of single particle excitations caused by one-body operators). The decay width is caused by the emission of particles into the continuum, and the collisional width by the coupling of the $1 p-1 h$ (one particle, one hole) states with nearby higher order states $(2 p-2 h, 3 p-3 h$, and so on) in the compound nucleus. The TDMF approach to nuclear giant resonances begins with a static Hartree-Fock calculation to define the ground state [4]. Excitations are then generated using an external perturbation in a time-dependent Hartree-Fock (TDHF) approach. Although collective, they stay generally in the linear regime of small amplitude motion. This allows one to linearize TDHF yielding the random phase approximation (RPA) $[5,6]$, which is a standard treatment of the excitation [7-10], sometimes reaching out to more sophisticated beyond-RPA techniques [11,12]. For nonsymmetric ground states as, e.g., in deformed nuclei, the conceptually 
simpler full TDHF treatment remains competitive with linearized versions and has become increasingly used [13-16]. For treating dynamics in the nonlinear regime, full TDHF is anyway compulsory. A similar situation is found also in cluster physics where the dynamical calculation of resonance excitations is called the time-dependent local-density approximation (TDLDA). For an extensive review (see [17]). The considerations of this paper are also relevant to these applications in atomic, molecular and cluster physics.

Using standard reflecting or periodic boundary conditions in TDHF or RPA results in an artificial discretization of the excitation spectrum. From a physical point of view, it means that one is able to describe the gross structure of spectra including the Landau width. A proper description of continuum structure, in particular of the decay width, requires absorbing or open boundary conditions. Several techniques have been employed for nuclear continuum RPA [18-23]. Only a subset of such methods is applicable to fully fledged TDHF, or TDLDA. The most widely used methods there are imaginary absorbing potentials, which have been applied in nuclear and atomic physics [14,25], and multiplicative masks applied to damp the wavefunctions [26]. These tools remove particles impinging on the surface of the box from further computation and thus approximate open boundaries. In the linear regime, they provide an interesting alternative to the often very elaborate continuum RPA techniques. It is the aim of this paper to investigate the quality and expense of the imaginary potential and multiplicative mask approach to absorbing boundary conditions in TDHF. For the linear response regime, we also compare with simulation of the continuum through driect smoothing techniques in the spectral analysis. For these calculations, we employ a onedimensional code (for radial motion) in order to use a huge variety of grid sizes for extensive tests. A confirmation is made of the broad usefulness of absorbing boundary conditions by looking also at a full three-dimensional (3D) TDHF calculation of a collision of two ${ }^{16} \mathrm{O}$ nuclei.

We shall, in Sec. II, briefly summarize the TDHF approach and its practical implementation. Our giant resonance test case is described in Sec. III, and the issues related to hard boundary conditions detailed in Sec. IV. In Sec. V, we make the analysis of absorption on the TDHF calculations, and in Sec. VI appears the study of smoothing in the spectral analysis, and a summary of all results for the linear case. Considerations of nonlinear breathing-mode excitations are given in Sec. VII, with a discussion of collisions in Sec. VIII. Section IX concludes.

\section{TIME-DEPENDENT HARTREE-FOCK}

Nuclear time-dependent Hartree-Fock calculations belong to the realm of the time-dependent density functional theory [27] where a many-body system is described in terms of single-particle wavefunctions $\varphi_{\alpha}$ and the local densities and currents build therefrom. The equations-of-motion are derived variationally from a given energy-density functional [4]. They read in quite general fashion

$$
i \hbar \dot{\varphi}_{\alpha}=\hat{h} \varphi_{\alpha}
$$

where $\hat{h}$ is the Hartree-Fock Hamiltonian which itself depends on the actual wavefunctions via the densities. It thus constitutes a nonlinear equation. Given an initial condition, $\left\{\varphi_{\alpha}(\mathbf{r}, t=0)\right\}$, Eq. (1) determines all $\varphi_{\alpha}$ at all times. This time-dependent mean-field theory naturally includes the Landau width and can, in principle, also describe the decay width. The Landau width comes from the underlying singleparticle spectrum, which is inherent in the Hartree-Fock calculations. We will always see a fragmentation in the spectrum due to the Landau width, but the correct calculation of the decay width will depend on the correct treatment of the outgoing particle flux at the boundary of the box. It is the decay width with which this paper is concerned.

There are several time stepping schemes for the solution of the TDHF (or TDLDA) equations. We use here the series expansion of the exponential evolution [28]. The TDHF equations are solved in practice in two steps. First, we perform a preliminary half-time step

$$
\widetilde{\varphi}_{\alpha}(\mathbf{r})=\exp (-i \hat{h}(t) \Delta t / 2) \varphi_{\alpha}(\mathbf{r}, t) .
$$

This is used to build an approximation to the mean-field $\tilde{h}$ at mid-time $t+\Delta t / 2$ in standard manner

$$
\left\{\widetilde{\varphi}_{\alpha}\right\} \Rightarrow \widetilde{\rho}, \quad \tilde{\mathbf{j}}, \ldots \Rightarrow \widetilde{h} .
$$

This then is used to perform the full step

$$
\varphi_{\alpha}(\mathbf{r}, t+\Delta t)=\exp (-i \tilde{h} \Delta t) \varphi_{\alpha}(\mathbf{r}, t) .
$$

The exponential is expanded in a power series and truncated at fourth order

$$
\exp (-i \hat{h} \Delta t) \approx \sum_{n=0}^{4} \frac{(-i \Delta t)^{n}}{n !} \hat{h}^{n}
$$

This choice has been found to be a sensible compromise between computational expense and stability, when combined with a suitable step size. In this study we have found a step size of $\Delta t=0.25 \mathrm{fm} / \mathrm{c}$ to be adequate when combined with the fourth-order Taylor Series expansion (2c). Unless stated otherwise, this time step is used in all calculations presented. The repeated application of the time evolution operator gives a time series of wavefunctions and derived quantities. The quality of the propagation is tested against norm and energy conservation (which applies for reflecting boundaries). The norm and energy are both conserved over the whole observation time within $10^{-5}$. This figure can be improved (or worsened) deliberately by adjusting the time step/or and number of terms retained in the Taylor Series expansion (2c).

\section{TEST SETUP}

As a practical application, the case of isoscalar monopole vibrations in ${ }^{16} \mathrm{O}$ is studied, using the Skyrme parameterization $\mathrm{SkM}^{*}$ [29]. Skyrme forces are effective nuclear interactions which reproduce the ground and certain excited state properties of nuclei across the periodic table [4]. They are generally fitted to basic ground state properties (masses, radii,...), and usually also including information from nuclear matter. Many of them also provide a good description of 
giant resonances, particularly for heavy nuclei while being less perfect for isovector modes in small nuclei $[9,30]$. Note that in the present paper we are not so much interested in a detailed comparison with experiment, as in an exploration of numerical issues. Therefore, we have chosen such a light nucleus to allow for widespread variations of the numerical conditions.

To create a monopole giant resonance, one must apply a suitable external perturbation. In all calculations presented here, the usual isoscalar monopole excitation operator

$$
F(r)=r^{2}
$$

is used to both create and measure the oscillations. The excitation is applied with a Gaussian time profile:

$$
\begin{aligned}
& V_{\text {ext }}(r, t)=\eta_{\text {ext }} F(r) f_{\text {ext }}(t), \\
& f_{\text {ext }}(t)=\exp \left(-\frac{\left(t-\tau_{0}\right)^{2}}{\tau_{\text {wid }}^{2}}\right) .
\end{aligned}
$$

with parameters

$$
\eta_{\mathrm{ext}}=0.01 \mathrm{MeV} r^{-2}, \quad \tau_{0}=5 \mathrm{fm} / \mathrm{c}, \quad \tau_{\text {wid }}=1 \mathrm{fm} / \mathrm{c} .
$$

This short time-span of the external perturbation simulates the brief impinging of a nuclear probe. It has a spectral width of about $200 \mathrm{MeV}$ and thus ensures that oscillations of all frequencies in the resonance range are excited with equal weight.

The spatial grid is set up with grid spacing $\Delta r=0.3 \mathrm{fm}$. The number of grid points, and with it the box size, is varied as a crucial parameter in the numerical study.

\section{GENERAL FEATURES WITHOUT ABSORPTION}

The isoscalar giant monopole resonance in ${ }^{16} \mathrm{O}$ lies at about $23 \mathrm{MeV}$ which is of order $10 \mathrm{MeV}$ above the continuum threshold. As such, the emitted nucleons have an average kinetic energy of about $10 \mathrm{MeV}$, corresponding to a mean velocity of $v \approx 0.14 \mathrm{c}$. The initial pulse (4) will thus release a bunch of nucleons which then travels along with that velocity towards the bounds of the box. Reflecting boundary conditions turn the bunch back to the nuclear center where it will reappear after a typical echo time

$$
T_{\text {echo }}=2 R_{\text {box }} / v \approx 14 R_{\text {box }} / \mathrm{c} .
$$

The echo and its dependence on the box should be visible in the recorded monopole signal. We can confirm this and see the effect of hard (=reflecting) boundary conditions by examining the echo delay time in the monopole response.

Figure 1 shows the amplitude of monopole oscillations as a function of time for various box sizes. In all three cases, the time signal is the same at the outset, since the outgoing flux did not have yet the time to reach the boundary. The amplitude of the oscillations decay in an exponential way, and would in principle die down to zero, except that there is that echo from reflux of nucleons reflected back from the bounds. The time it takes for this to happen depends on the radius,

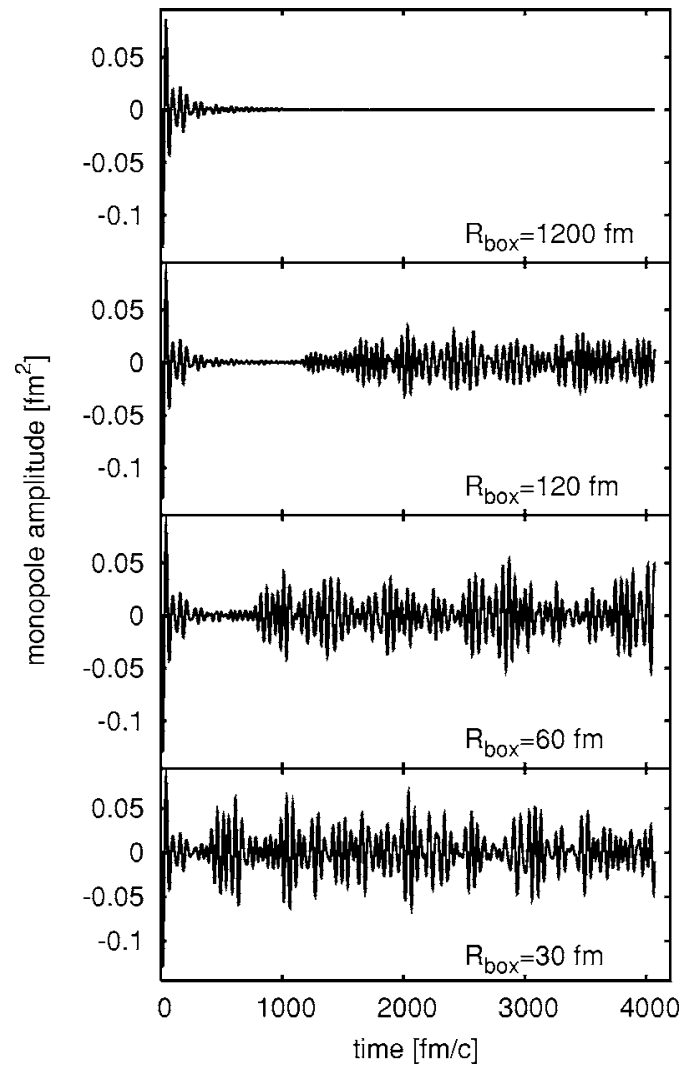

FIG. 1. Monopole oscillation in the time domain for several box sizes.

$R_{\text {box }}$ of the box as estimated in Eq. (5). Therefore, for $R_{\text {box }}$ $=30,60$, and $120 \mathrm{fm}$, delays of 400, 800, and $1600 \mathrm{fm} / \mathrm{c}$ are the expected values in broad agreement with Fig. 1. Since the outgoing particle flux contains components traveling at different velocities, once can see that the shape of the returning echo looks different for different box sizes, and in particular is the more stretched the larger the box.

This echo is, of course, artificial. It allows standing waves to set up in the box, which discretize the spectrum [13]. In principle one could allow the box size to grow without limit, and obtain a closer and closer approximation to the continuum result, a strategy which is discussed in Sec. VI B. One can see from the top frame in Fig. 1 that the echo may be delayed arbitrarily, delivering arbitrary resolution. But one is bound by limits of computational time, and a finite box size must be used; for example, the $1200 \mathrm{fm}$ box of Fig. 1 is too large for a three-dimensional calculation. One can estimate the necessary size of a (reflecting) box according to the wanted energy resolution. An unperturbed (by the echo signal) observation time of $T_{\text {obs }}$ allows an energy resolution of about $\pi / T_{\text {obs }}$, and using Eq. (5) one can establish an estimate in terms of box size for the considered test case

$$
\delta \omega_{\mathrm{echo}}=\frac{\pi}{T_{\mathrm{obs}}}=\frac{v \pi}{2 R_{\mathrm{box}}} \approx \frac{0.1 c}{R_{\mathrm{box}}} .
$$

For example, a $T_{\text {obs }} \approx 4000 \mathrm{fm} / \mathrm{c}$ observation yields a resolution of $\delta \omega_{\text {echo }} \approx 0.14 \mathrm{MeV}$ and requires a box of $R_{\mathrm{box}} \approx 300 \mathrm{fm}$. 


\section{ABSORBING BOUNDS}

When the flux reaches the edge of the box, it should in principle continue out as if the boundary were not there. For a finite box, this effect can be simulated using some kind of absorbing boundary. The absorption of the outgoing wave, or suppression of the echo will never be quite perfect, and the study of the optimal ways of applying these techniques is discussed here. The method for quantifying the quality of an absorbing algorithm will in this section simply be the amplitude of the echo.

The two methods of simulating the absorbing boundary conditions considered in this study are the use of an imaginary potential and a masking function. For the first option, a linear optical potential is used [14]

$$
V_{\mathrm{opt}}=-i W_{\mathrm{opt}} \theta\left(r-R_{\mathrm{box}}\right)\left(r-R_{\mathrm{box}}\right) .
$$

This potential is added to the mean-field Hamiltonian $\hat{h}$ in all time steps. Note that the margin carrying the optical potential is added outside the original numerical box, i.e., outside $R_{\text {box }}$. A certain number of absorbing points $N_{\text {abs }}$ are used while keeping the same grid spacing. The quality of absorption will depend on the size of this absorbing margin. The masking function, on the other hand, supplements the time step (2) by one masking step

$$
\varphi_{\alpha} \rightarrow \varphi_{\alpha} \cos \left(\frac{\pi}{2} \frac{r-R_{\mathrm{box}}}{R_{\mathrm{abs}}}\right)^{p}
$$

applied after the final sub-step (2b). This masking function involves the radial distance $r=|\mathbf{r}|$ and is appropriate for the radial $1 \mathrm{D}$ problem treated here. Other geometries require other masks, see, e.g., [31]. The $R_{\mathrm{abs}}=N_{\mathrm{abs}} \Delta r$ is the added absorbing margin and $N_{\text {abs }}$ is the number of grid points over which the masking is applied. The $\Delta r$ is the same radial grid spacing as used in the interior.

Both have much in common. In fact, one can map the masking function into an equivalent optical potential. For simplicity, we assume a mere first-order expansion of the exponential step, i.e., $1-i \delta t \hat{h}$. In this case the masking operation can be achieved by employing an effective optical potential

$$
V_{\mathrm{opt}}^{(\mathrm{eff})}=-\frac{i}{\delta t}\left[1-\cos \left(\frac{\pi}{2} \frac{r_{\nu}-R_{\mathrm{box}}}{R_{\mathrm{abs}}}\right)^{p}\right] \approx-i \frac{p}{\delta t}\left(\frac{\pi}{2} \frac{r_{\nu}-R_{\mathrm{box}}}{R_{\mathrm{abs}}}\right)^{2} .
$$

It is obvious that the step size $\delta t$ plays a role: The shorter the time step the stronger the optical potential. It is the combination $p / \delta t$ which determines the effective damping strength.

Figure 2 shows an example of monopole oscillations computed with absorbing bounds of type (8) compared with a result from reflecting bounds using the same box size. One sees a much reduced and somewhat delayed echo. The delayed echo is explained by the fact that the absorbing bounds are more efficient for fast particles such that the reflected wave has a higher content of slow velocities. Moreover, one sees also that the absorption is by no means perfect in that a visible amount of back-flow is still seen. We now need to work out optimal damping conditions for both methods by

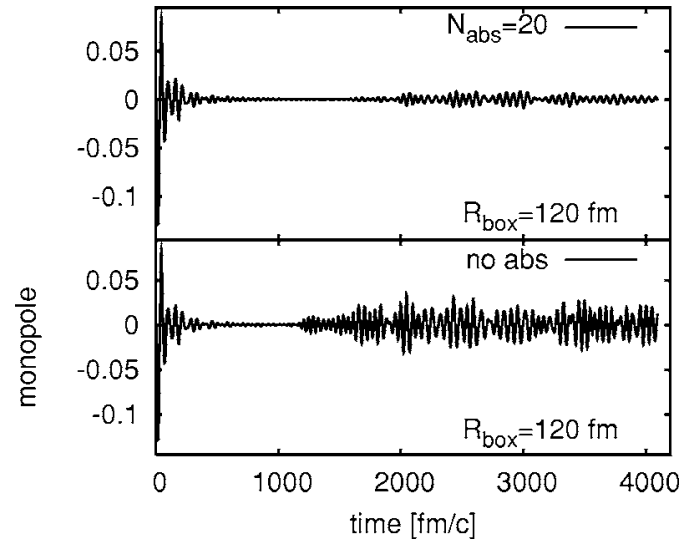

FIG. 2. Monopole oscillation amplitude in the time domain. The lower panel shows the case of hard reflecting boundary conditions, the upper panel shows a masking absorption with 20 extra points, and a power $p=0.04$.

systematically varying the parameters. In order to simplify the analysis, we characterize the quality of absorption by one number: The maximum amplitude of the echo.

Figure 3 shows the results of series of calculations for the maximum echo amplitude as a function of the damping strength $W_{\text {opt }}$ for the optical potential or the power $p$ for the masking function. Two different numbers of extra absorbing grid points, $N_{\text {abs }}$, are used and the size of the time step is also varied in case of the masking function (lower panel). Clearly in either prescription the larger absorbing margin performs better than the smaller, and the best echo reduction is about the same. With $N_{\mathrm{abs}}=20$, the reduction in amplitude approached one order of magnitude. In the $N_{\mathrm{abs}}=100$ case,
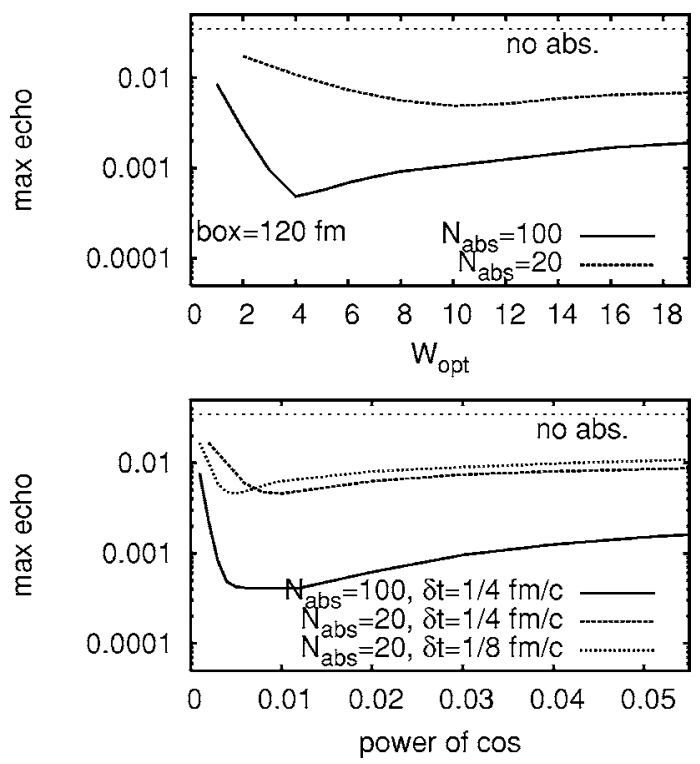

FIG. 3. Maximum echo in monopole amplitude as a function of the number of absorbing points $N_{\text {abs }}$ for the two types of boundary condition considered. Upper panel: Results using the optical potential (7). Lower panel: Results using the masking function (8). The faint dotted horizontal line show the amplitude without absorbing bounds for comparison. 
around two orders of magnitude reduction in the echo amplitude was found.

In both cases, the absorption shows a weak minimum in dependence on the damping parameter. Too small damping leaves an appreciable quantity of flux reflected back, whereas too large absorption causes reflection from the steep optical potential at the beginning of the absorbing region. A best compromise is found in between. It is seen that, in the case of an imaginary potential, the optimum strength $W_{\text {abs }}$ depends on the number of absorbing points used. For the masking function with given time step $\delta t$, the preferred value for $p$ is quite insensitive to $N_{\text {abs }}$, although altering the time step moves the minimum which can be understood from the mapping (9) where the effective damping turns out to be $p / \delta t$. In both cases, the choice of the optimum working point is not awfully critical. One can (and one should) calibrate the method once for a broad set of applications within a given numerical setup.

\section{TEST CASE LINEAR RESPONSE}

\section{A. The principles of spectral analysis}

The most important physical observable associated with giant resonances is the strength function. In the case of the isoscalar giant monopole resonances considered in this work, this corresponds to the electric monopole strength associated with a transition from the nuclear ground state to the giant resonance, as a function of energy. The strength function of an observable $\hat{F}$ is defined in general as

$$
S(E)=-\frac{1}{\pi} \lim _{\delta \rightarrow 0} \operatorname{Im}\left\langle 0\left|\hat{F} \frac{1}{H-E+i \delta} \hat{F}\right| 0\right\rangle,
$$

where Im stands for the imaginary part. It can be computed with TDHF and spectral analysis as follows [24]: One starts the dynamical evolution at $t=0$ from the mean-field ground state, applies the short pulse (4), and measures the $F$-signal over time

$$
F(t)=\sum_{\alpha}\left\langle\varphi_{\alpha}(t)|\hat{F}| \varphi_{\alpha}(t)\right\rangle .
$$

At the end, one performs a Fourier transformation into the frequency domain

$$
\tilde{F}(\omega)=\int_{0}^{\infty} d t e^{i \omega t} F(t)
$$

and similarly for the time profile of the excitation function $f(t) \rightarrow \widetilde{f}(\omega)$. The strength function is then finally

$$
S_{F}(\omega)=-\frac{1}{\pi} \operatorname{Im}\left\{\frac{\tilde{F}(\omega)}{\tilde{f}(\omega)}\right\} .
$$

Note that the spatial form of the function $F(r, t)$ is to be exactly the same in the excitation (4) and in the analysis (11). In the test case here, we use the isoscalar monopole (3).

The actual TDHF calculations stop, of course, at some finite final time $T_{\text {fin }}$ and the data are sampled on a discrete equidistant mesh $t_{\nu}=\nu \delta t$. We thus replace in practice the Fourier integral by the Fourier series

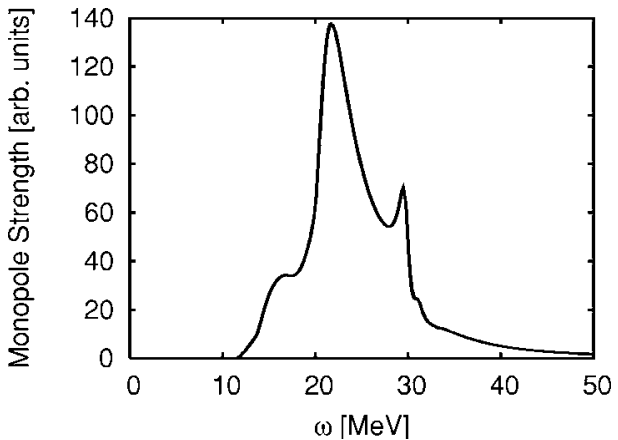

FIG. 4. The continuum strength function for the isoscalar monopole resonance in ${ }^{16} \mathrm{O}$ with Skyrme force $\mathrm{SkM}^{*}$. A $1200 \mathrm{fm}$ box was used, and an observation time of $8192 \mathrm{fm} / \mathrm{c}$, so that the boundary was not reached by the outgoing flux.

$$
\begin{gathered}
\widetilde{F}\left(\omega_{k}\right)=\frac{\delta t}{2} F(0)+\sum_{\nu=1}^{N_{\text {fin }}} \delta t e^{i \omega_{k} t} \nu F\left(t_{\nu}\right), \\
\omega_{k}=k \frac{\pi}{T_{\text {fin }}}, \quad k \in\left\{0, \ldots, N_{\text {fin }}\right\},
\end{gathered}
$$

where $T_{\text {fin }}=N_{\text {fin }} \delta t$. This defines the strength on a mesh $\omega_{k}$ in frequency space. The maximal spectral resolution achievable on the mesh is $\hbar \pi / T_{\text {fin }}$. For example, a simulation time of $T_{\text {fin }}=8000 \mathrm{fm} / \mathrm{c}$ yields a spectral mesh spacing of $0.07 \mathrm{MeV}$. An example of such a strength function is shown in Fig. 4. It is taken from the Fourier transform of a signal in which a $1200 \mathrm{fm}$ box was used along with an observation time of $8192 \mathrm{fm} / \mathrm{c}$ (the first half was shown in the uppermost panel in Fig. 1), leading to a situation in which the outgoing flux has not yet reached the boundary. The pattern looks already quite realistic as compared to typical experimental strength distributions, although it is missing the additional contribution from the collisional width which is beyond the capabilities of a mean-field theory, but which is not so large for the isoscalar modes, particularly the monopole [3]. Most structures are very soft indicating a typical decay wdth of about 1 to $2 \mathrm{MeV}$. Note the sharp spike at $30 \mathrm{MeV}$. This is a bound $1 p h$ state (the $1 s \rightarrow 2 s$ transition) embedded in the continuum. This figure, computed very carefully on a huge numerical box and sampled over a long time interval, will serve as a benchmark for our further considerations.

\section{B. Filtering in the time domain}

The spectral analysis thus far is unambiguous if the signal has fully died down at the end, i.e., $F\left(T_{\text {fin }}\right)=0$. In more practical calculations, however, one uses smaller boxes and makes use of absorbing boundary conditions. Since the absorption is not perfect, one obtains finite signals throughout due to the echoes. The resulting forced cutoff at $T_{\text {fin }}$ with finite signals at the end of the interval gives rise to artefacts in the spectra [32]. One has to apply some windowing to produce smooth strength distributions. We use a windowing in the time domain multiplying the signal with a smooth cut-off function as 
TABLE I. The relationship between the power of the cosine mask applied to the time signal, and the equivalent folding width $\Delta \omega$.

\begin{tabular}{lcccccccc}
\hline \hline \multicolumn{1}{c}{$N_{\text {filt }}$} & 2 & 8 & 32 & 128 & 512 & 2048 & 8192 \\
\hline FWHM $/ T_{\text {fin }}$ & 1 & $\frac{1}{2}$ & $\frac{1}{4}$ & $\frac{1}{8}$ & $\frac{1}{16}$ & $\frac{1}{32}$ & $\frac{1}{64}$ \\
& & & & & & & & \\
& & & & & & & \\
FWHM (fm/c) & 16384 & 8193 & 4096 & 2048 & 1024 & 512 & 256 \\
$\Delta \omega(\mathrm{MeV})$ & 0.05 & 0.1 & 0.2 & 0.4 & 0.8 & 1.6 & 3.2 \\
\hline \hline
\end{tabular}

$$
F(r, t) \rightarrow F(r, t) \cos \left(\frac{t \pi}{2 T_{\text {fin }}}\right)^{N_{\text {filt }}}
$$

and with even cut-off powers $N_{\text {filt }}$. This filtering function has the property that it dampens the effective signal to approach a zero final value rapidly in a most smooth manner (vanishing derivatives up to order $N_{\text {filt }}-1$ ). It approaches a Gaussian for large values of $N_{\text {filt }}$. The variation of the filtering strength $N_{\text {filt }}$ allows to vary the full width at half maximum (FWHM) of the effective signal. One has approximately

$$
\mathrm{FWHM}=T_{\text {fin }} \sqrt{\frac{2}{N_{\text {filt }}}} .
$$

The smoothing of the spectra by forced premature extinction of the signal has the price that one loses some spectral resolution. It now becomes

$$
\Delta \omega \frac{2 \pi}{\text { FWHM }}=\sqrt{2 N_{\text {filt }}} \frac{\pi}{T_{\text {fin }}} .
$$

As we will see, the deliberate sacrifice of resolution is necessary to provide a reasonable interpretation of the spectra in case of no or insufficient absorption. We, therefore, prefer to call $\Delta \omega$ a smoothing width. An example for filtering strength and related FWHM as well as smoothing widths is given in Table I. In practice, we find that mainly the final FWHM counts in the achieved spectral pattern. It is then more efficient to use a low filtering strength of typically $N_{\text {filt }}=2$ or 4 with correspondingly short simulation time $T_{\text {fin }}$.

Figure 5 shows strength functions in the absence of any absorbing boundary conditions. Each panel stands for one particular choice of box size and compares different folding strengths $N_{\text {filt }}$, and accordingly different folding widths as tabulated in Table I. In the case of the smallest spectral smoothing $\Delta \omega=0.05 \mathrm{MeV}$, one sees the sharp discrete spectrum that occurs in the absence of absorbing bounds. These occur at the frequencies of the RPA eigenmodes in the phase space given by the finite box. As the size of the box is increased, as seen in the different panels of Fig. 5 the increased density of states in the phase space is reflected in the increased number of eigenmodes appearing in accordance with the spectral density of single particle states in a spherical potential well.

Enhancing the filtering strength, i.e., increasing the smoothing width $\Delta \omega$, changes the spectrum to a smother pattern. Insufficient filtering leaves some oscillations about the wanted continuum strength. Such oscillations could easily be misinterpreted as detailed fragmentation structures in the spectra. One has to make sure that the smoothing width $\delta \omega$ is larger than the (artificial) level spacing in a finite spherical well which we we can read off in the energy range around $20 \mathrm{MeV}$ approximately as $\Delta E=3$ and $0.8 \mathrm{MeV}$ for the box sizes $R_{\mathrm{box}}=15$ and $60 \mathrm{fm}$, respectively. For the smaller box size of $15 \mathrm{fm}$, only the the largest smoothing width of $\Delta \omega=3.2$ guarantees wiping out all artifacts. With this largest spreading, the strength is close to the continuum result, but lacks the finer points of its structure as, e.g., the spike at $30 \mathrm{MeV}$. This is due to the insufficient information obtained from the small phase space available to the discretized calculation in small boxes, compared to the continuum calculation.

In the case of the larger, $60 \mathrm{fm}$, box shown in the right two panels of Fig. 5 , the $\Delta \omega=0.8 \mathrm{MeV}$ spreading is already sufficient to smooth the discretized spectrum to resemble the continuum case since the discrete peaks are closer in energy than in the $15 \mathrm{fm}$ case.

\section{Competition between filtering and absorption}

We thus have introduced two basically different ways to simulate the smooth spectral distribution of strengths above emission threshold, the absorbing boundary conditions and strong spectral filtering. We will investigate in this section the combination of both methods, their cooperation or competition. Having ascertained the optimum values for the parameters $W_{\text {abs }}$ and $p$ associated with the linear absorbing potential and masking function, respectively (see Fig. 3), we select these optimal values in each case, and study the effect of varying the number of absorbing points, $N_{\mathrm{abs}}$, the box size, $R_{\text {box }}$ and the smoothing width $\Delta \omega$.

Figure 6 repeats the analysis of Fig. 5, except that an extra 20 absorbing points carrying the optical potential are used
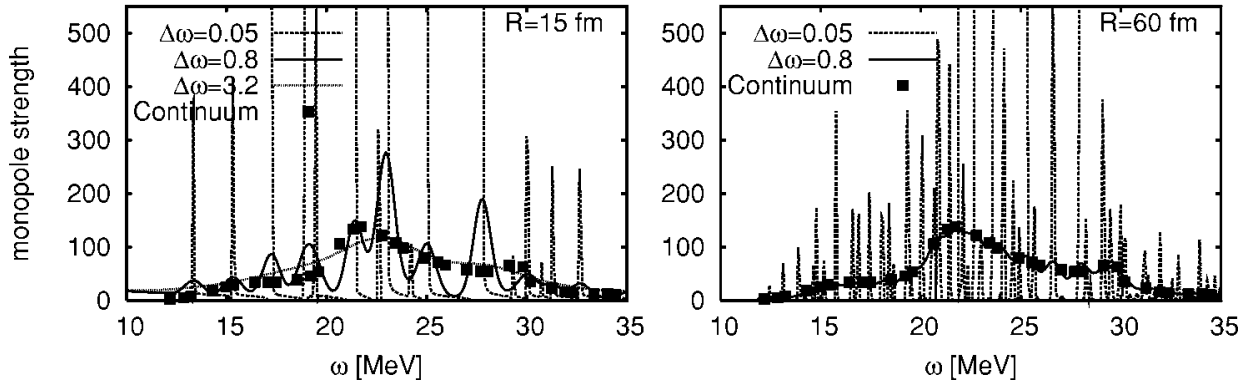

FIG. 5. Strength functions for calculations without absorption. Each panel collects results for a single box size, as indicated, for various folding widths $\Delta \omega$. Note that the $\Delta \omega=0.05$ results go well beyond the $y$-axis range. 

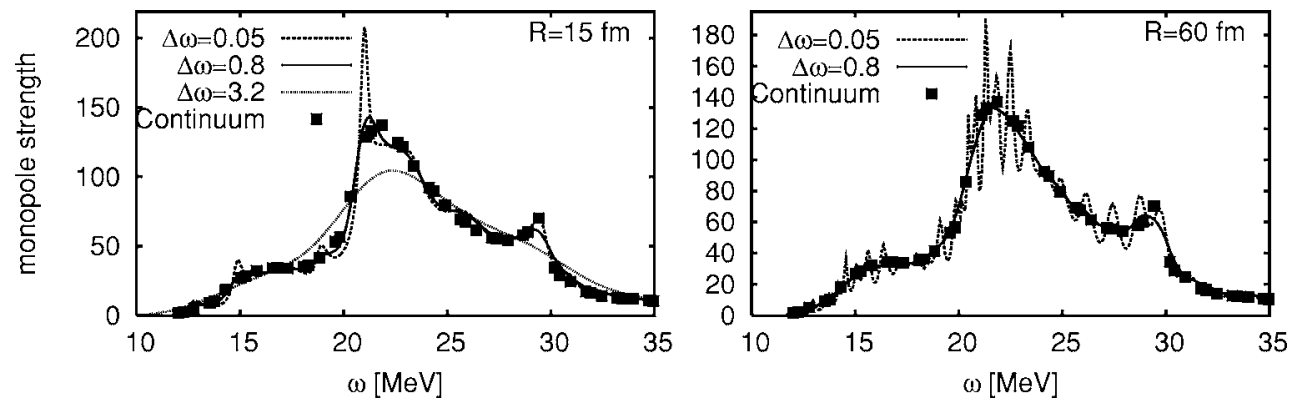

FIG. 6. Strength functions as Figure 5, but with $N_{\text {abs }}=20$.

beyond the end of the box. One immediately sees that the sharp peaks of the lightly filtered spectra $(\Delta \omega=0.05 \mathrm{MeV})$ are dramatically reduced and smoothed out, somewhat like the $\Delta \omega=0.8 \mathrm{MeV}$ curve in Fig. 5 without the imaginary absorbing potential. This is not surprising since the effect of the imaginary potential is to make the eigenmodes of the box complex, and hence damped. In spite of the dramatic reduction, there remain still sizeable oscillations which leave the pattern far from the goal. The small smoothing of $0.8 \mathrm{MeV}$ improves a lot, but still leaves some artifacts for the $20 \mathrm{fm}$ box. Only the largest smoothing finally helps here, however, at the price of overriding true structure in the spectrum, similar to with the case of no absorption. For the $60 \mathrm{fm}$ box, the moderate smoothing with $0.8 \mathrm{MeV}$ provides almost perfect agreement with the wanted spectrum.

We have seen up to now that larger smoothing widths allow approaching smooth spectra more easily. Too large a width, however, wipes out wanted physical structures. It seems that the largest acceptable smoothing is $0.8 \mathrm{MeV}$ for the present test case. We will have a closer look at that choice. Choosing a fixed $\Delta \omega=0.8 \mathrm{MeV}$, Fig. 7 explores the effect of varying the number of absorbing points, $N_{\mathrm{abs}}$, for two different box sizes. Without absorption, only even larger boxes would deliver a satisfying result. Increasing absorption allows dealing with smaller boxes and yet yields acceptable results. With $N_{\mathrm{abs}}=20$ a box of $60 \mathrm{fm}$ suffices, and with $N_{\text {abs }}=100$, we can step down to a box of $30 \mathrm{fm}$ (not shown here) while reproducing the essential structures of the spectrum. The step from $N_{\mathrm{abs}}=0$ to $N_{\mathrm{abs}}=20$ constitutes a substantial gain in expense as we can give up 200 true grid points (from $R_{\text {box }}=120 \mathrm{fm}$ down to $60 \mathrm{fm}$ ) while adding only 20 points for the absorbing margin. The next step to $N_{\mathrm{abs}}=100$ does not gain as much, since we give up 100 true points and add another 80 absorbing points.

\section{Summary of times and energies}

In this section, we summarize briefly the key quantities for the analysis of resonance spectra in the particle continuum. Table II gives an overview. The average velocity of emitted nucleons $\bar{v}$ depends on the average resonance energy $E_{\text {res }}$ relative to the emission threshold $E_{\text {thresh. It determines }}$ the decay width $\Gamma_{\text {decay }}$, the level spacing $\delta \omega_{\text {box }}$ of the (artificial) discretization of the box, and the echo time $T_{\text {echo }}$. Note that $\delta \omega_{\text {box }}$ accounts for the level spacing of the single nucleon states in the pseudo-continuum. The actual spacing between $1 p h$ states looks much denser due to the overlay of states related different hole states. But the spectral resolution is set by the density of continuum states as quantified in terms of $\delta \omega_{\text {box }}$. The decay width, in turn, sets the scale for the wanted spectral resolution $\Delta \omega$ of the analysis. This, on the other hand, has to overcome the pseudo-discretization of the continuum in case that we work without absorbing bounds and rely fully on the spectral smoothing by windowing. The scales are then set by

$$
\Gamma_{\text {decay }}>\Delta \omega>\delta \omega_{\text {box }} .
$$

The $\Delta \omega$ is tuned by the simulation time $T_{\text {fin }}$ in combination with the filtering strength $N_{\text {filt }}$. As finally only the net FWHM counts, it is most efficient to use a low $N_{\text {filt }}=2$ or 4 and to thus have the shortest possible $T_{\text {fin }}$. This holds with and without absorbing bounds. The second inequality in condition (16) applies to the case without absorbing bounds. It determines the minimum box size $R_{\text {box }}$ required for that resolu-
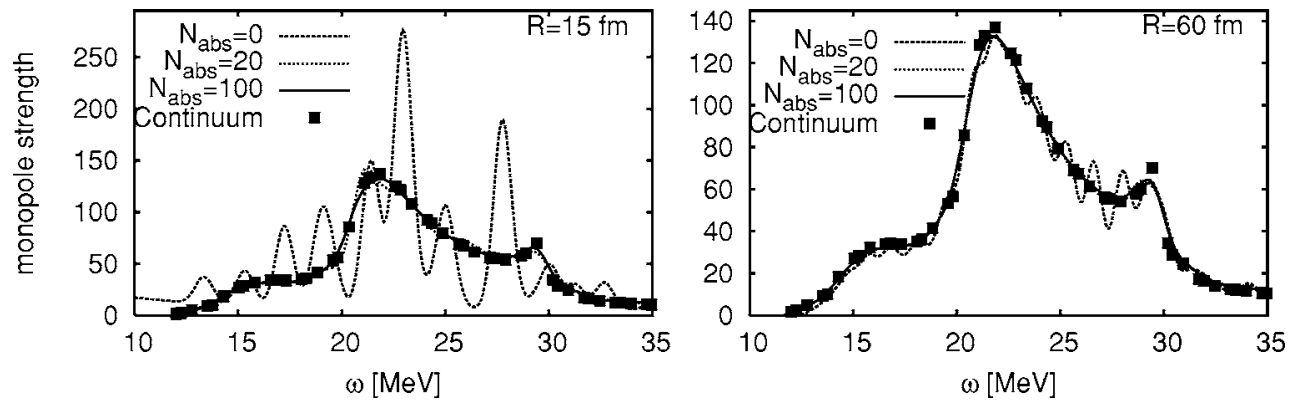

FIG. 7. Strength functions with and without absorbing bounds. Each panel collects results for a given box size, as labeled, for a range of $N_{\text {abs }}$. 
TABLE II. Compilation of key quantities in estimating strength functions in the continuum. The second column contains the general formal relation while the third column exemplifies it for the present test case.

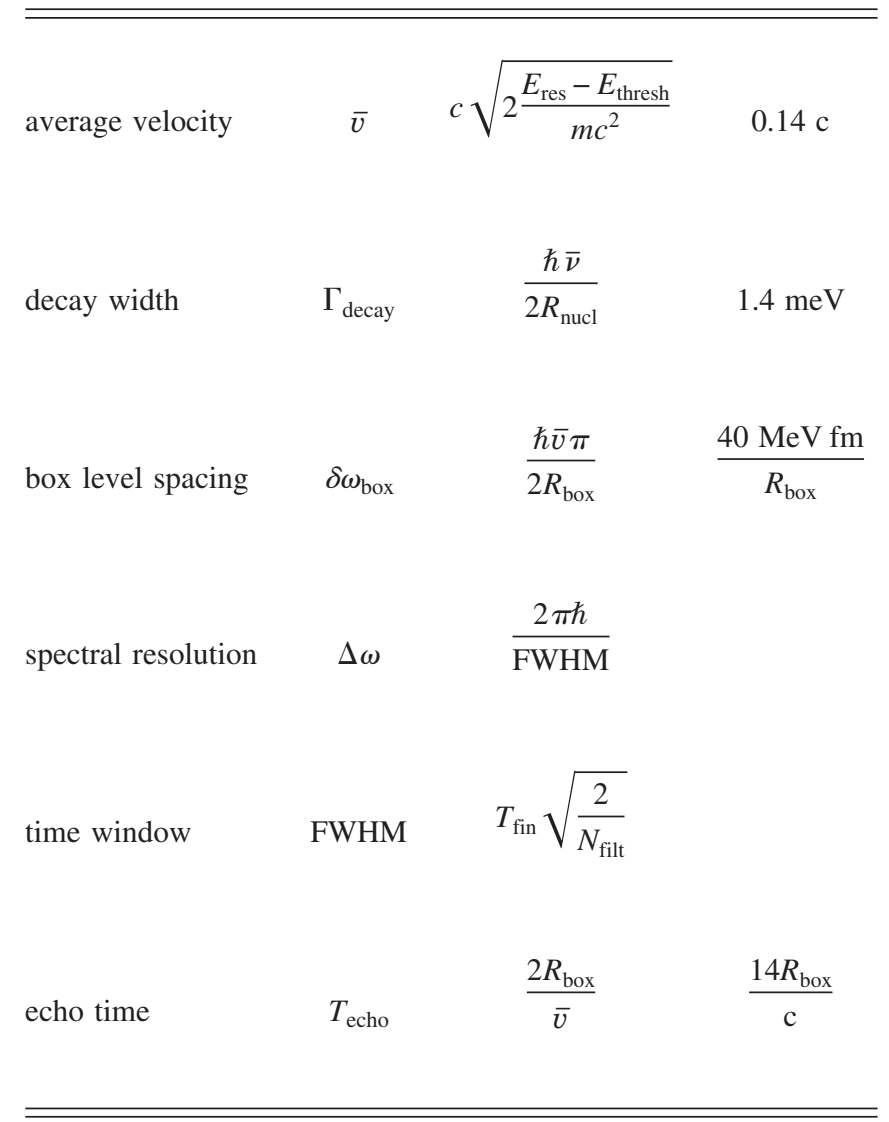

tion. Invoking absorbing boundary conditions allows to use smaller box sizes than required by the above condition. A reduction by a factor of two was found to be conceivable with $N_{\text {abs }}=20$ extra grid points in the absorbing margin, and a factor four with $N_{\mathrm{abs}}=100$. The conceptually simplest, but computationally most expensive, way to produce a clean spectrum is to chose the box so large that the echo time $T_{\text {echo }}$ exceeds safely the decay time of the resonance and to perform straightforward spectral analysis with the then cleanly damped time signal.

Note that this analysis is based on producing as authentic a spectrum from the TDHF equations as possible. This, by definition, omits the width due to collisions. Inclusion of the collision width will actually soften the above criteria, since it contributes extra broadening and helps wash out artefacts.

\section{TESTS IN THE NONLINEAR DOMAIN}

A major motivation for development and application of absorbing boundary conditions comes from the realm of nonlinear mean-field dynamics. For the original atomic physics case see, e.g., $[25,26]$. The quest for good absorption persists also for heavy-ion dynamics. Violent collisions will always be accompanied by nucleon emission. One has to prevent those emitted nucleons from falling back onto the reaction zone. We will investigate the performance of absorbing

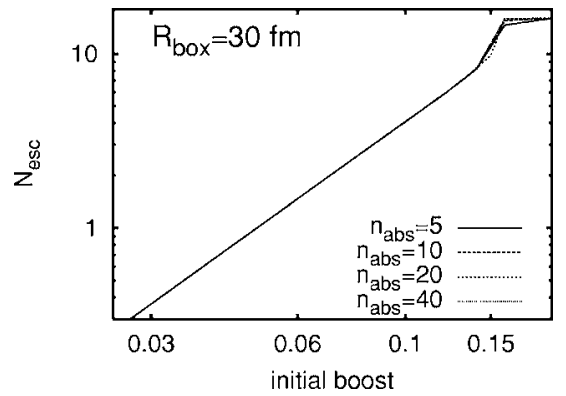

FIG. 8. Total number of emitted nucleons after excitation by an extremely short pulse (boost) of varying strength. The boost strength is given in terms of radial momentum in units of $\mathrm{fm}^{-2}$. Test case is ${ }^{16} \mathrm{O}$ computed with the force Skyrme $\mathrm{M}^{*}$ in a radial box of $30 \mathrm{fm}$. The number of absorbing points $N_{\mathrm{abs}}$ is varied as indicated.

bounds in the nonlinear regime. Our test cases are the large amplitude oscillation of ${ }^{16} \mathrm{O}$ as induced by short force pulses typically occuring in hefty collisions, and an actual collision between two ${ }^{16} \mathrm{O}$ nuclei. Many observables in the nonlinear domain are related to particle emission as, e.g., angular distribution, kinetic energy distribution, net ionization [17]. We consider as one such observable the ionization from strong breathing oscillations.

We simulate a very short force pulse by initial excitation with a radial boost $\varphi \rightarrow \exp \left(i p_{\text {boost }} r^{2}\right) \varphi$. The radial momentum $p_{\text {boost }}$ regulates the strength of the excitation. The energy imprinted to the system through the boost will be removed to a large extent by particle emission. We measure the average total emission as the loss of the total normalization $N_{\text {esc }}(t)$ $=\int d^{3} r \rho(r, t)-N(t=0)$. One can also resolve in detail the particle loss from each single particle state separately as $N_{\alpha}(t)$ $=\int d^{3} r\left|\varphi_{\alpha}(r, t)\right|^{2}$ : With some combinatorial analysis, one can compute from that the detailed probability $P(n, t)$ to find the state with $n$ emitted particles $[33,34]$. We find in our tests again the equivalence between the imaginary potential method (7) and the masking function technique (8). Thus we will show only results for the imaginary potentials.

Figure 8 shows the total emission finally achieved as function of the boost strength for a variety of absorbing margins. The results do not depend so sensitively on the size of the margin for this global observable. This shows that gross effects in the nonlinear regime can very well be estimated with rather inexpensive small absorbing margins, which is common practice in most of these calculations.

Figure 9 shows the more detailed emission probabilities finally achieved, i.e., $P(n, t \rightarrow \infty)$. Here we see slightly more sensitivity. The smallest margin shows small but visible deviations. The three larger margins have already well converged. That is still much more robust than in the extremely critical linear response regime.

Figure 10 shows as another detail the total emission as function of time. Here we see again somewhat more sensitivity than in the global final value of Fig. 8. The qualitative pattern are still reproduced for any margin. In particular, we see that all margins are capable of final absorption and thus suppressing the unwanted rebounce. But a more detailed analysis will always require the larger margins. 


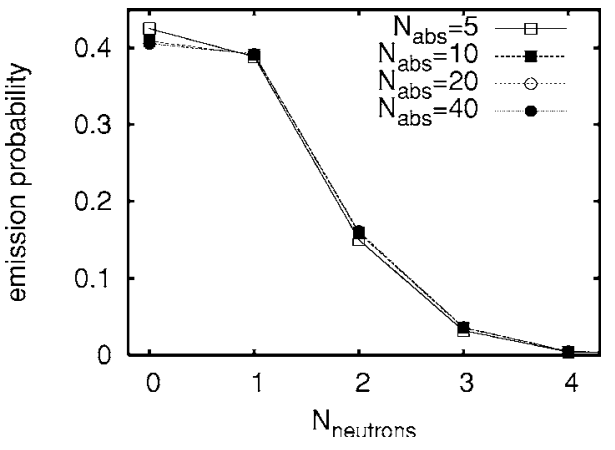

FIG. 9. The probability distribution for emitting a certain number of neutrons after excitation by an extremely short pulse (boost) of varying strength. The boost strength is given in terms of radial momentum in units of $\mathrm{fm}^{-2}$. The test case is ${ }^{16} \mathrm{O}$ computed with the force Skyrme $\mathrm{M}^{*}$ in a radial box of $30 \mathrm{fm}$. The number of absorbing points $N_{\text {abs }}$ is varied as indicated.

In any case, we find that well adjusted absorbing bounds are a very efficient and reliable method in the domain of nonlinear dynamics. They are compulsory to avoid artefacts from reflected particle flow. On the other hand, rather affordable small margins suffice in that case. This regime is thus much different from the linear response where rather large margins are required for quantitative success and where alternatives (although not cheap either) exist.

\section{NUCLEAR COLLISIONS}

To highlight the importance of absorbing bounds in nonlinear applications of TDHF, and to accentuate their wide application, we have also studied collisions. As a test we again use the ${ }^{16} \mathrm{O}$ nucleus, this time colliding two such nuclei and examining the conservation of energy with and without absorption. For this we use a full three-dimensional timedependent Hartree-Fock code in which two separate ${ }^{16} \mathrm{O}$ nuclei are initialized, given a boost which sets them toward each other, and are allowed to collide.

In time-dependent Hartree-Fock the kinetic energy of relative motion of two clusters is not an immediately avail-

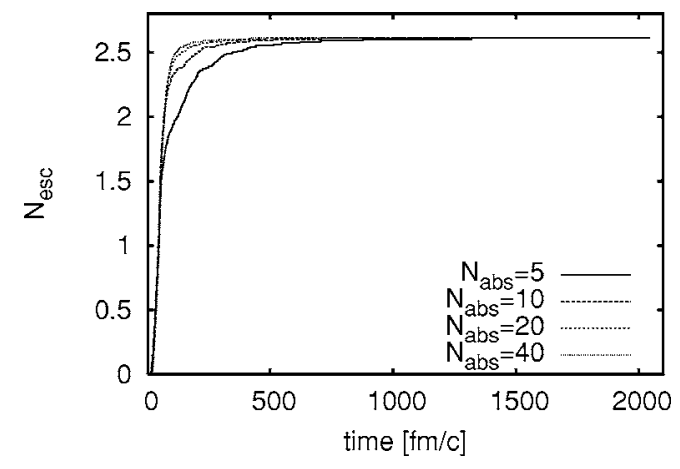

FIG. 10. The detailed time-evolution of the total number of emitted nucleons after excitation by an extremely short pulse (boost) with initial radial momentum of $0.08 \mathrm{fm}^{-2}$. Test case is ${ }^{16} \mathrm{O}$ computed with the force Skyrme $\mathrm{M}^{*}$ in a radial box of $30 \mathrm{fm}$. The number of absorbing points $N_{\mathrm{abs}}$ is varied as indicated.

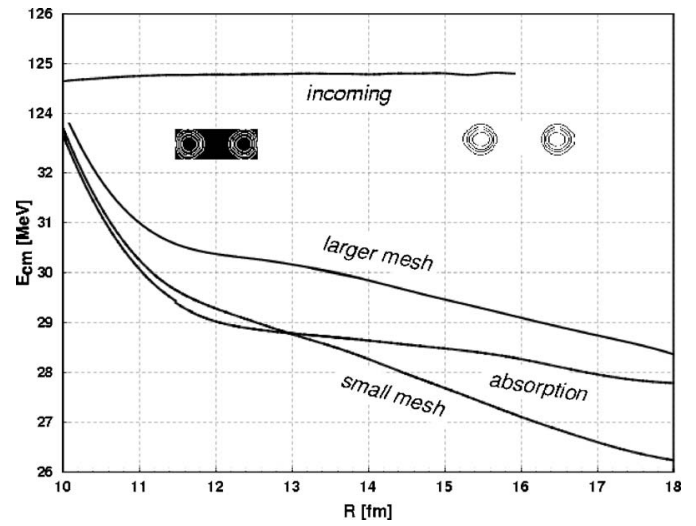

FIG. 11. Center-of-mass energy for the collision of two ${ }^{16} \mathrm{O}$ nuclei. The energy before collision is shown, as are three cases for post-collision $\mathrm{CM}$ energy, according to the cases described in the text.

able quantity. Instead, it must be deduced using a two-body analysis of the time-dependent density distribution.

The code first tries to determine whether the system has separated into two fragments. For this purpose it calculates the principal axes of the mass quadrupole tensor of the combined system. Then it examines the density along the axis of maximum quadrupole moment to find whether it shows the characteristics of two maxima separates by a low-density region. The point of lowest density along this line then defines a dividing plane perpendicular to this axis, and two fragments are assumed to exist on both sides of this plane. Calculating the centers of mass of each fragment yields a new straight line connecting them, which is used to repeat the process. This is iterated until the definition of the fragment centers of mass and the dividing plane have stabilized. The principal result of this analysis are the fragment masses and charges $M_{i}, Q_{i}, i=1,2$, the separation distance $R$ of the fragments, and the relative velocity $\dot{R}$ calculated from the values of $R$ at two successive time steps (for noncentral collisions the angular momentum of c.m. motion can also be calculated, but this is of no concern for the present discussion).

For head-on collisions the energy of relative motion can then be calculated from the simple formula

$$
E_{\mathrm{cm}}(R)=\frac{1}{2} \frac{M_{1} M_{2}}{M_{1}+M_{2}} \dot{R}^{2}+\frac{Q_{1} Q_{2}}{R},
$$

where the Coulomb energy is approximated by the expression for two point charges.

Figure 11 shows a calculation of the relative-motion energy $E_{\mathrm{cm}}(R)$ for ${ }^{16} \mathrm{O}+{ }^{16} \mathrm{O}$ at an initial c.m. energy of $125 \mathrm{MeV}$. An impression of the physical situation is given by the small insets. The two nuclei are initialized at a distance of $16 \mathrm{fm}$, so that they have a distace of at least $5 \mathrm{fm}$ to travel towards each other before significant interaction sets in. The quantity $E_{\mathrm{cm}}$ for this "incoming" part of the reaction is shown in the upper curve. The initialization reduces the value of $E_{\mathrm{cm}}$ to about $124.80 \mathrm{MeV}$, but this value stays remarkably constant with changes below $0.02 \mathrm{MeV}$ down to 
$R=12 \mathrm{fm}$. This shows that the numerical description of free propagation through the grid and the two-body analysis work quite well.

To add to the picture, it should be mentioned that during the full collision calculation (not only the initial phase) the total energy decreases by $0.02 \mathrm{MeV}$, while the total particle number fluctuates by about 0.01 nucleons.

In the final stages of reseparated fragments, however, the situation appears quit different. The lower three curves in Fig. 11 show that $E_{\mathrm{cm}}$ does not stay constant, as it should. They correspond to different numerical and physical conditions, which do not lead to any differences in the "incoming" part of the collision.

The curve labeled "small mesh" refers to a calculation on a grid with $24 \times 24 \times 32$ points with a spacing of $1 \mathrm{fm}$. The boundary conditions for the wave functions are periodic, since we use an fast Fourier transform (FFT) method for representing the derivatives. The time step was $0.2 \mathrm{fm} / \mathrm{c}$. As seen by the conservation properties above and by the agreement for the incoming phase, this yields sufficient accuracy for the pure solution of the TDHF equations.

Here we see that for this case $E_{\mathrm{cm}}$ drops by $3 \mathrm{Mev}$ for $R$ going from 12 to $18 \mathrm{fm}$. This implies an equivalent uncertainty in the determination of the final energy, which is perhaps not unaceeptable but certainly annoying.

The possibilty that this effect arises from artefacts in the Coulomb energy can be excluded. The Poisson equation is solved with the method of [35] using a double-sized grid which ensures a correct handling of the long-range behavior. The monopole approximation used for the estimate (17) has been tested by including also the quadrupole interaction energy, whereby (17) turned out to perform sufficiently precise.

Since Coulomb can be ruled out, the remaining possibility for explaining the effect is direct interference of the wave functions with their periodic images, and in this respect the emission of free nucleons again would be the prime candidate, since the bound states should decay sufficiently rapidly. The two additional curves in Fig. 11 show results of tests in this direction: Making the mesh larger by increasing the dimensions to $32 \times 32 \times 44$ (with grid spacing remaining at $1 \mathrm{fm}$, curve labeled "larger mesh") changes the absolute magnitude of the effect but the continuing drop in $E_{\mathrm{cm}}$ remains about the same.

On the other hand, adding an absorbing boundary (curve labeled "absorption") of 4 cells in each direction reduces the variation in $E_{\mathrm{cm}}$ considerably: It is close to $1 \mathrm{MeV}$ now. It appears that the emission of nucleons and their spurious reinterference with the dynamics inside the computational grid is a problem for the simulation of heavy-ion collisions as well.

In the future a careful balance will have to be chosen between the need for accuracy in the final c.m. energy and computational efficiency, since adding an absorbing layer means a considerable increase in computing time. Further studies concerning the energy and mass dependence are clearly required.

\section{CONCLUSIONS}

We have studied two widely used techniques for simulating absorbing boundary conditions (imaginary potentials and masking functions) in the time-dependent mean-field approach, using as test cases the description of nuclear giant resonances and large-amplitude nuclear collective motion associated with significant breakup probabilities. Straightforward calculation in a finite box with the standard reflecting boundary conditions yields unwanted echoes in the signal due to nucleon current reflected from the bounds an reappearing at the nuclear site. This can induce unpredictable side-effects in large amplitude dynamics. In the analysis of the giant resonance strength function, these echoes artificially "quantize" the spectra in accordance with the discrete spectrum in a finite simulation box, thus missing the smooth spectral distributions as they emerge from the decay width in the particle continuum.

A conceptually simple, but extremely costly, solution is to use huge boxes such that the echoes do not show up within the observation time (which in turn determines the resolution of the computation). A broad palette of elaborate techniques has been developed to establish continuum solutions in the linear regime (continuum RPA). We have investigated two of them which are easily applicable also in large-scale and large-amplitude situations. These are absorbing boundary conditions which aim to remove outgoing flow. The two options, the absorbing potential and masking function, were found to be about equally effective, and able to reduce echoes by two orders of magnitude for only moderate computational cost. It is important that reasonable values of the parameters associated with the absorbing potential and masking function are used to ensure efficient absorption. It is found, however, that the remaining small echoes still give rise to visible oscillations in the spectra.

For computing spectra in the linear regime, a simpler and more direct way of smoothing the spectra is achieved by windowing in the spectral analysis. It was realized by filtering of the given signal in the time domain. That again brings up a payoff between resolution and expense. The smoothing has to override the average spectral spacing of the finite box. Rather large boxes are needed to maintain sufficient sensitivity in order to see the wanted physical structures in the spectra. It was found finally that a judiciously chosen mix of moderately absorbing bounds with moderate spectral smoothing provides the most efficient scheme.

In the analysis of large amplitude motion for the breathing mode, the absorbing bound technique worked very well, and the relevant observables, such as number of emitted nucleons, was rather insensitive to the details of the absorption used. It is clear that the computation of spectra in the continuum near the emission threshold is probably the most demanding application for absorbing boundary conditions. As little as a remaining percent of reflected particles can still yield a visible perturbation of the spectra. Other applications, for which the absorbing were designed originally, are much more forgiving. For example, the outgoing flow of emitted particle can be easily computed with a precision of a few percent which is fully satisfying for that observable and others of similar nature (as, e.g., angular distributions). However, further applications to resonance spectra call for improvement of absorbing bounds.

While our work concentrated on applications to breathing mode oscillations, our study of nuclear collisions again high- 
lights the importance of absorbing boundary conditions. Without them, the conservation of energy is badly violated. Their inclusion improves matters, and indicates that their application is widespread in TDMF approaches to diverse physical scenarios.

\section{ACKNOWLEDGMENTS}

This work was funded in part by the Bundesministerium für Bildung und Forschung (BMBF), Project Nos. 06 ER 808 and 06 ER 124, and in part from EPSRC under Grant No. GR/S96425/01.
[1] M. N. Harakeh and A. Van der Woude, Giant Resonances (Oxford University Press, Oxford, 2001).

[2] G. F. Bertsch, P. F. Bortignon, and R. A. Broglia, Rev. Mod. Phys. 55, 287 (1983).

[3] P.-G. Reinhard, H. L. Yadav, and C. Toepffer, Nucl. Phys. A 458, 301 (1986).

[4] Michael Bender, Paul-Henri Heenen, and P.-G. Reinhard, Rev. Mod. Phys. 75, 121 (2003).

[5] D. J. Rowe, Nuclear Collective Motion (Methuen, London, 1970).

[6] P. Ring and P. Schuck, The Nuclear Many-Body Problem (Springer-Verlag, New York, 1980).

[7] G. Bertsch, Phys. Rev. Lett. 31, 121 (1973).

[8] S. Shlomo and G. Bertsch, Nucl. Phys. A 243, 507 (1975).

[9] P.-G. Reinhard, Ann. Phys. 1, 632 (1992).

[10] G. F. Bertsch and R. Broglia, Oscillations in Finite Quantum Systems (Cambridge University Press, Cambridge, 1994).

[11] D. Lacroix and Ph. Chomaz, Phys. Rev. C 60, 064307 (1999).

[12] S. Ayik, D. Lacroix, and Ph. Chomaz, Phys. Rev. C 61, 014608 (2000).

[13] P. D. Stevenson, M. R. Strayer, J. Rikovska Stone, and W. G. Newton, Int. J. Mod. Phys. E 13, 181 (2004).

[14] T. Nakatsukasa and K. Yabana, Phys. Rev. C 71, 024301 (2005).

[15] D. Vretenar, A. V. Afanasjev, G. A. Lalazissis, and P. Ring, Phys. Rep. 409, 101 (2005).

[16] J. A. Maruhn, P.-G. Reinhard, P. D. Stevenson, J. R. Stone, and M. R. Strayer, Phys. Rev. C 71, 064328 (2005).

[17] P.-G. Reinhard and E. Suraud, Introduction to Cluster Dynamics (Wiley, Berlin, 2003).

[18] M. Cavinato, M. Marangoni, P. L. Ottaviani, and A. M. Saruis,
Nucl. Phys. A 373, 445 (1982).

[19] N. Van Giai, P. F. Bortignon, F. Zardi, and R. A. Broglia, Phys. Lett. B 199, 155 (1987).

[20] M. Bianchetti, M. R. Quaglia, G. Colo, P. M. Pizzochero, R. A. Broglia, and P. F. Bortignon, Phys. Rev. C 56, R1675 (1997).

[21] K. Hagino and H. Sagawa, Nucl. Phys. A 695, 82 (2001).

[22] E. A. Moukhal, V. A. Rodin, and M. H. Urin, Phys. Lett. B 447, 8 (1999).

[23] T. Nakatsukasa and K. Yabana, RIKEN Rev. 39, 96 (2001).

[24] F. Calvayrac, P.-G. Reinhard, and E. Suraud, Ann. Phys. (N.Y.) 255, 125 (1997).

[25] Kenneth C. Kulander, Phys. Rev. A 35, R445 (1987).

[26] J. L. Krause, K. J. Schafer, and K. C. Kulander, Phys. Rev. A 45, 4998 (1992).

[27] P.-G. Reinhard and E. Suraud, in Metal Clusters, edited by W. Ekardt (Wiley, New York, 1999), p. 121.

[28] H. Flocard, S. E. Koonin, and M. S. Weiss, Phys. Rev. C 17, 1682 (1978).

[29] J. Bartel, P. Quentin, M. Brack, C. Guet, and H.-B. Hakansson, Nucl. Phys. A 386, 79 (1982)

[30] P.-G. Reinhard, Nucl. Phys. A 649, 305c (1999).

[31] F. Calvayrac, P.-G. Reinhard, E. Suraud, and C. A. Ullrich, Phys. Rep. 337, 493 (2000).

[32] W. H. Press, S. A. Teukolsky, W. T. Vetterling, and B. P. Flannery, Numerical Recipes in C: The Art of Scientific Computing (Cambridge University Press, New York, 1992).

[33] C. A. Ullrich, PhD thesis, Würzburg, 1995.

[34] F. Calvayrac, A. Domps, P.-G. Reinhard, E. Suraud, and C. A. Ullrich, Eur. Phys. J. D 4, 207 (1998).

[35] J. W. Eastwood and D. R. K. Brownrigg, J. Comput. Phys. 32, 24 (1979). 